\title{
Searching for Low-Mass Supermassive Black Holes
}

\author{
Michele Cappellari ${ }^{1}$, Francesco Bertola ${ }^{1}$, Enrico M. Corsini ${ }^{1}$, \\ José G. Funes, S.J. ${ }^{1}$, Alessandro Pizzella ${ }^{2} \&$ Juan C. Vega Beltrán ${ }^{3}$ \\ ${ }^{1}$ Dipartimento di Astronomia, Università di Padova, Italy \\ ${ }^{2}$ European Southern Observatory, Santiago, Chile \\ ${ }^{3}$ Osservatorio Astronomico di Padova, TNG, Italy
}

It has become generally accepted that most or possibly all ellipticals and bulges of spirals harbor supermassive black holes in their center (see Ho 1998 for a recent review).

We have constructed a model for a thin gaseous disk orbiting in the combined potential of a central point-like mass embedded in a diffuse component mimicking the stellar disk. We have used this model to reproduce the peculiar bidimensional shape of the emission lines in a sample of early type disk galaxies, observed with ground based telescopes (e.g. Fig. 1). In this way it has been possible to deduce the presence of nuclear mass concentrations of the order of $1 \times 10^{9} \mathrm{M}_{\odot}$ in four objects (Bertola et al. 1998).

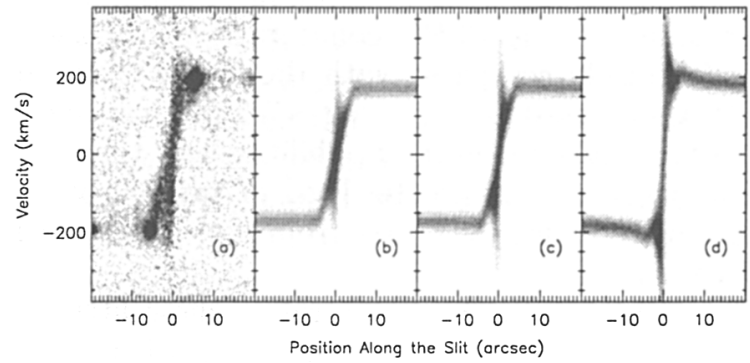

Figure 1. (a): The $\mathrm{H} \alpha$ emission line, observed along the major axis of NGC 2179, after subtraction of the stellar continuum. (b), (c) and (d): Models of the NGC $2179 \mathrm{H} \alpha$ line [shown in the same scale of panel (a)] obtained with different point-like central masses. (b): $M_{\bullet}=2 \times 10^{8}$ $\mathrm{M}_{\odot}$. (c): $M_{\bullet}=1 \times 10^{9} \mathrm{M}_{\odot}$ corresponding to our best-fit model. (d): $M_{\bullet}=5 \times 10^{9} \mathrm{M}_{\odot}$.

In some of the observed galaxies (e.g. NGC 5064) the rotation curve does not show any peculiar bidimensional shape, but rises almost linearly from $v=0$ at $r=0$ up to an almost constant value at larger radii (Fig. 2a). By applying our modeling technique, taking into account the observational parameters of our ground based observations, we have estimated that in these cases the central mass, if present, has to be lower than $5 \times 10^{7} \mathrm{M}_{\odot}$ (Fig. 2b).

In Fig. 3 we present a simulation of an HST spectrum obtained with STIS (grating G750M with the 52 " $\times 0.2$ " slit) for the same gaseous disk modeled in 
Fig. 2b. It is apparent that the much higher spatial resolution of HST would still allow us to detect the central perturbation due to the $5 \times 10^{7} \mathrm{M}_{\odot}$ black hole adopted for this model.

We think that disk galaxies like NGC 5064 are good candidates to harbor low-mass supermassive black holes. This example shows that the combination of ground based observations, with properly equipped telescopes, and followup HST observations could be an efficient way to explore the low end of the black-hole mass function.

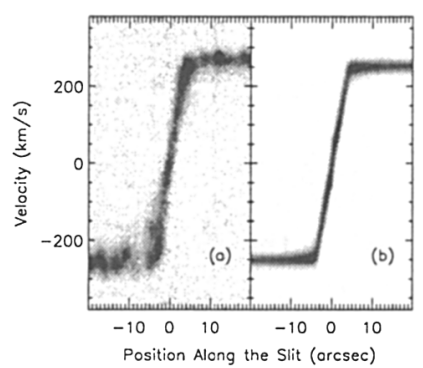

Figure 2. (a): The $\mathrm{H} \alpha$ emission line, observed with the ESO 3.6$\mathrm{m}$ telescope equipped with CASPEC echelle spectrograph, along the major axis of NGC 5064, after subtraction of the stellar continuum. (b): Model of the NGC $5064 \mathrm{H} \alpha$ line obtained with the highest pointlike central mass which can be added without significantly disturbing the general shape of the line $\left(M_{\bullet}=5 \times 10^{7} \mathrm{M}_{\odot}\right)$.

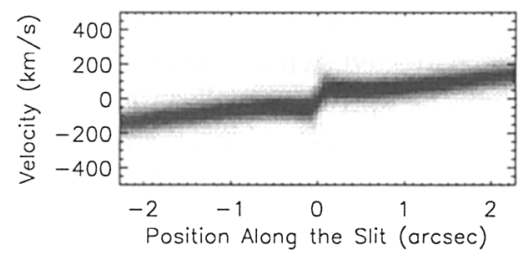

Figure 3. Simulation of an HST spectroscopic observation with STIS (grating G750M with the 52 " $\times 0.2$ " slit) for the same gaseous disk modeled in Fig. 2b. The reciprocal dispersion $\Delta v$ is $\sim 8 \times$ smaller (better) in the case of the ground based observation of Fig. $2 b$, but the spatial resolution is $\sim 24 \times$ better for the STIS observation. Note that only the very inner region of the profile is shown in this Figure.

\section{References}

Bertola, F., Cappellari, M., Funes, J.G., Corsini, E.M., Pizzella, A., \& Vega Beltrán, J.C. 1998, ApJL, in press [astro-ph/9807075]

Ho, L.C. 1998, in Observational Evidence for Black Hole in the Universe, ed. S.K. Chakrabati (Dodrecht: Kluver Academic Publishers), in press [astro$\mathrm{ph} / 9803307]$ 